\title{
Nurturing breakthroughs Lessons from complexity theory
}

\section{Journal Article}

\section{Author(s):}

Sornette, Didier

Publication date:

2008

Permanent link:

https://doi.org/10.3929/ethz-b-000008397

\section{Rights / license:}

In Copyright - Non-Commercial Use Permitted

\section{Originally published in:}

Journal of Economic Interaction and Coordination 3(2), https://doi.org/10.1007/s11403-008-0040-8 


\title{
Nurturing breakthroughs: lessons from complexity theory
}

\author{
D. Sornette
}

Published online: 18 April 2008

(C) Springer-Verlag 2008

\begin{abstract}
A general theory of innovation and progress in human society is outlined, based on the combat between two opposite forces (conservatism/inertia and speculative herding "bubble" behavior). We contend that human affairs are characterized by ubiquitous "bubbles", which involve huge risks which would not otherwise be taken using standard cost/benefit analysis. Bubbles result from self-reinforcing positive feedbacks. This leads to explore uncharted territories and niches whose rare successes lead to extraordinary discoveries and provide the base for the observed accelerating development of technology and of the economy. But the returns are very heterogeneous and very risky. In other words, bubbles, which are characteristic definitions of human activity, allow huge risks to get huge returns over large scales. We refer to and summarize a large bibliography covering our research efforts in the last decade, which present the relevant underlying mathematical tools and a few results involving positive feedbacks, emergence, heavy-tailed power laws, outliers/kings, the problem of predictability and the illusion of control.
\end{abstract}

\section{Introduction}

An ambitious ultimate goal for science is to support and inform good policy decision making. In order to address this challenge, it seems to me that one needs first to embrace the larger question of what is driving a human being, a human groups or a human society. Only then can policy decision making identify and aim correctly its targets. My perception is that these questions can now be addressed with a fresh perspective, informed and inspired by science. Indeed, the scientific enterprise is now offering insights on many of the questions in social sciences that were before only the

D. Sornette $(\varangle)$

Department of Management, Technology and Economics, ETH Zurich,

Kreuzplatz 5, 8032 Zurich, Switzerland

e-mail: dsornette@ethz.ch 
realm of normative approaches or philosophical discourses. Via novel experimental procedures, new technology and new conceptual insights, science can now help address from an evidence-based approach such questions as: what is happiness (Osberg and Sharpe 2005; Revkin 2005; Kahneman et al. 2006; Reichhardt 2006; Griffin 2007)? What is driving human beings? Why do we cooperate?

Here, I can only address a subset of these important questions. My discussion is organized in four short parts.

1. The first one recognizes that most systems are punctuated by rare but large events which often dominate their organization. The progress of science and technology is no exception, as innovations, discoveries, blockbusters are exceptional events in their impact. This statement can be quantified by heavy-tailed distributions.

2. Complementing the first part which emphasizes the statistics of the impacts of innovations, the second part focuses on a classification of their time dynamics, by presenting a simple classification of the dynamics of complex systems in terms of the interplay between endogenous and exogenous shocks. This allows us to separate the impact of external influences from the role played by the constitution of the inner structure in understanding and predicting blockbusters. This is applied to different critical events in economics and the social sciences taken as proxies of innovation dynamics: social unrest shocks, internal downloads, dialog in email traffic, the dynamics of commercial sale in response to advertisement or to word-of-mouth, volatility clustering and shocks in financial markets, dynamics of exploits and patches following disclosures of software vulnerability, movie blockbusters, the dynamics of YouTube video sharing, and so on.

3. The third part documents the phenomenon that I coin "breakdown of the human Galilean invariance principle", namely that humans being bored by steady state tend to act to develop intermittent accelerating outcomes. As a consequence of their individual actions aggregated at the collective level, one can observe superexponential acceleration of their processes punctuated by corrections and crashes. The mathematical description of these processes emphasizes the importance of positive feedbacks. Based on this idea, I propose that "bubbles" are generic results of collective human activities and that they seem to be not only inherently associated with human societies but are also a vehicle of giant leaps in progress.

4. Lastly, the question of control and management of complex systems is alluded to by stressing the phenomenon of "illusion of control" and its consequence for practical policies.

The last section concludes.

\section{Heavy-tail distribution of breakthroughs and blockbusters}

Many studies report evidences of positive economic benefits derived from basic research (Martin Ben et al. 1996; NAS 1997). In certain areas such as biotechnology, semi-conductor physics, optical communications (Ehrenreich 1995), the impact of basic research is direct while, in other disciplines, the path from discovery to applications is full of surprises. As a consequence, there are persistent uncertainties in the quantification of the exact economic returns of public expenditure on basic research. 
This gives little help to policy makers trying to determine what should be the level of funding and how should it be implemented (see however the remarkable study of Hollingsworth and Hollingsworth 2000).

Some industries, such as the pharmaceutical and movie industries, are characterized by the occurrence of "block-busters," i.e. remarkably successful products with exceptional sales much larger than the typical product. Determining how exceptional are these block-busters is an important question for firm strategy and economies of scale. Taking the movie industry as a proxy because the data is unambiguous, plentiful and of good quality, Sornette and Zajdenweber (1999) have shown that the distribution of gross revenues of Hollywood movies from theatres resulting from the top box office 100 is stable over twenty years and is well-described by a power law distribution with exponent $\alpha$ approximately equal to 1.5 . Specifically, the probability density function $p(S)$ of the random variable defined as the gross revenue $S$ of a given movie is of the form $p(S) \sim 1 / S^{1+\mu}$, with $\mu \approx 1.5$. In this case, the expectation $\mathrm{E}[S]=\int S p(S) d S$ is finite but the variance of $S$ which involves the second order moment $\int S^{2} p(S) d S$ is infinite. Grabowski and Vernon $(1990,1994)$ constructed a discounted present value per new chemical entity and divided the drugs in decile in descending order, leading to a value distribution compatible with a power law distribution of the tail with exponent approximately equal to 2/3 (Sornette 2002a; Sornette, unpublished), for which neither the mean not the variance exist. Scherer (1998) has studied the distribution of royalties from US University patent portfolios, of the quasi-rents from marketed pharmaceutical entities and the stock market returns from three large samples of high-technology venture start-ups. The tails of the distributions are again compatible with a power law distribution with exponent less than 1 but there is a noticeable curvature when going to small returns. D. Harhoff and F.M. Scherer (personal communication) have studied a sample of approximately 800 high-value patents and find that the distribution most closely approximates a log normal while the power law hypothesis is strongly rejected when using the whole sample. The issue whether the extreme tail of the distribution of returns from innovation is asymptotically a power law with a small exponent is thus a delicate statistical problem. This is not specific to this domain of application, see for instance (Sornette 2004).

Sornette and Zajdenweber (1999) have suggested that these uncertainties on what should be the relevant policy on research support have a fundamental origin to be found in the interplay between the intrinsic "fat tail" power law nature of the distribution of economic returns of innovations, characterized by a mathematically diverging variance, and the stochastic character of discovery rates. In the regime where the cumulative economic wealth derived from research is expected to exhibit a long-term positive trend, they show that strong fluctuations blur out significantly the short-time scales: a few major unpredictable innovations may provide a finite fraction of the total creation of wealth. In such a scenario, any attempt to assess the economic impact of research over a finite time horizon encompassing only a small number of major discoveries is bound to be at best unreliable and at worst misleading.

In the Kuhnian view of how science works (Kuhn 1970), periods of "normal science" are interrupted by revolutions. If discovery "sizes" are indeed distributed according to a power law distribution, it is natural to wonder if Kuhn was only half-correct: it seems 
possible that there is no such thing as "normal science", and that science instead evolves through a succession of "revolutions" of all sizes. This idea has been put forward in another context by M. Buchanan (1996). Accordingly, history only takes notice of the really huge "revolutions"-quantum theory and relativity, for example-even though there are less significant others going on all the time. As a signature of this idea, there should be some kind of Gutenberg-Richter law for ideas - a power law distribution of their impact, as found by Redner (1998) and Dieks and Chang (1976) for the impacts of scientific publications.

This suggests to bring the problem of research economic benefits into the growing basket of natural and societal processes characterized by extreme behavior. They range from large natural catastrophes such as volcanic eruptions, hurricanes and tornadoes, landslides, avalanches, lightning strikes, catastrophic events of environmental degradation, to the failure of engineering structures, social unrest leading to large-scale strikes and upheaval, economic drawdowns on national and global scales, regional power blackouts, traffic gridlock, diseases and epidemics, etc. These phenomena are extreme events that occur rarely, albeit with extraordinary impact, and are thus completely under-sampled and thus poorly constrained. They seem to result from self-organising systems which develop similar patterns over many scales, from the very small to the very large. There is an urgency to assimilate in our culture and policy that we are embedded in extreme phenomena. Our overall sense of continuity, safety and confort may just be an illusion stemming from our myopic view. Let us unleash the battle of giants between extraordinary discoveries and extreme catastrophes.

\section{Interplay between endogenous and exogenous shocks (endo-exo): from commercial sales to happiness}

\subsection{Motivations}

Self-organized criticality, and more generally, complex system theory contend that out-of-equilibrium slowly driven systems with threshold dynamics relax through a hierarchy of avalanches of all sizes. Accordingly, extreme events are seen to be endogenous (Bak and Paczuski 1995; Bak 1996) in contrast with previous prevailing views. In addition, the preparation processes before large avalanches are almost undistinguishable from those before small avalanches, making the prediction of the former basically impossible (see Sornette $2002 \mathrm{~b}$ for a discussion). But, how can one assert with $100 \%$ confidence that a given extreme event is really due to an endogenous self-organization of the system, rather than to the response to an external shock? Most natural and social systems are indeed continuously subjected to external stimulations, noises, shocks, sollications, forcing, which can widely vary in amplitude. It is thus not clear a priori if a given large event is due to a strong exogenous shock, to the internal dynamics of the system organizing in response to the continuous flow of small sollicitations, or maybe to a combination of both. Adressing this question is fundamental for understanding the relative importance of self-organization versus external forcing in complex systems and for the understanding and prediction of crises. 
This leads to two questions:

1. Are there distinguishing properties that characterize endogenous versus exogenous shocks?

2. What are the relationships between endogenous and exogenous shocks?

Actually, the second question has a long tradition in physics. It is at the basis of the interrogations that scientists perform on the enormously varied systems they study. The idea is simple: subject the system to a perturbation, a "kick" of some sort, and measure its response as a function of time, of the nature of the sollicitations and of the various environmental factors that can be controlled. In physical systems at the thermodynamic equilibrium, the answer is known under the name of the theorem of fluctuation-dissipation, sometimes also refered to as the theorem of fluctuationsusceptibility (Stratonovich 1992). In a nutshell, this theorem relates quantitatively in a very precise way the response of the system to an instantaneous kick (exogeneous) to the correlation function of its spontaneous fluctuations (endogenous). An early example of this relationship is found in Einstein's relation between the diffusion coefficient $D$ of a particle in a fluid subjected to the chaotic collisions of the fluid molecules and the coefficient $\eta$ of viscosity of the fluid (Einstein 1905, 1956). The coefficient $\eta$ controls the drag, i.e., response of the particle velocity when subjected to an exogenous force impulse. The coefficient $D$ can be shown to be a direct measure of the (integral of the) correlation function of the spontaneous (endogenous) fluctuations of the particle velocity, leading to its inverse proportionality to the drag coefficient $\eta$.

In out-of-equilibrium systems, the existence of a relationship between the response function to external kicks and spontaneous internal fluctuations is not settled (Ruelle 2004). In many complex systems, this question amounts to distinguishing between endogeneity and exogeneity and is important for understanding the relative effects of self-organization versus external impacts. This is difficult in most physical systems because externally imposed perturbations may lie outside the complex attractor which itself may exhibit bifurcations. Therefore, observable perturbations are often misclassified. It is thus interesting to study other systems, in which the dividing line between endogenous and exogenous shocks, while not necessarily clearer, occurs in different ways, so that the diversity of examples and case studies may provide more hints on how to model complex physical systems.

We should stress that we are saying much more that the triviality that systems experience both internal and external processes. The key point of the endogenousexogenous question (which we will refer to as "endo-exo" for short) is to realize that one can learn on the internal organization of a system by studying its response to an external kick, and vice-versa, understanding the endogenous dynamics provides a basis for predicted the response to an external perturbation. This is the essence of the fluctuation-susceptibility theorem mentioned above. A similar relationship between the endogenous organization and the response to exogenous shocks has been recently documented quantitatively in the following systems:

1. Commercial successes (progressive reputation cascade versus the result of a well orchestrated advertisement) (Sornette et al. 2004a; Deschatres and Sornette 2005),

2. Financial crashes (external shocks versus self-organized instability) (Johansen and Sornette 2001, 2007; Sornette 2003a,b), 
3. Intermittent bursts of financial volatility (external shocks versus cumulative effects of news in a long-memory system) (Sornette et al. 2003, 2004b),

4. Social unrests (triggering factor or rotting of social tissue) (Roehner et al. 2004; Roehner 2007),

5. Earthquakes with their foreshocks/aftershocks patterns quantified by the inverse Omori and the direct Omori laws (Helmstetter and Sornette 2003; Helmstetter et al. 2003),

6. View dynamics of videos on YouTube.com for nearly 5 million videos (Crane and Sornette 2008).

In all these systems, the response function to an external kick is found to be a power law whose exponent is related to the exponent of the power law describing the decay of a spontaneous endogenous fluctuations. This endo-exo relationship provides a precise embodiment of our proposal.

We propose that similar relationships connect the response function to an exogenous shock and the relaxation from a spontaneous endogenous, suggesting the need to reassess the real origin of the observed shocks/catastrophes in the following systems:

- Biological extinctions such as the Cretaceous/Tertiary KT boundary (meteorite versus extreme volcanic activity (Deccan traps) versus self-organized critical extinction cascades),

- immune system deficiencies (external viral/bacterial infections versus internal cascades of regulatory breakdowns) (Sornette et al. 2007),

- cognition and brain learning processes (role of external inputs versus internal self-organization and reinforcements),

- discoveries (serendipity versus the outcome of slow endogenous maturation processes),

- the aviation industry recession (9/11/2001 terrorist attack versus structural endogenous problems),

- recovery after wars (internally generated (civil wars) versus imported from the outside) and so on.

The relevance of both internal and external shocks has in fact been widely investigated for many kinds of complex systems, including those listed here. For ecosystems, for instance, Holling and his colleagues (Holling et al. 2002) have published extensively about "adaptive cycles." The question of catastrophic impacts versus self-organized criticality in evolution has been the subject of intense argument in the literature over recent years (Bak 1996). In these contexts, our endo-exo approach is based on the novel derivation of a quantitative relationship between the relaxation dynamics following an exogenous shock compared to that after a spontaneous endogenous fluctuation, as explained in the previously mentioned examples (Sornette et al. 2004a; Deschatres and Sornette 2005; Johansen and Sornette 2001, 2007; Sornette 2003a,b; Sornette et al. 2003, 2004b; Roehner et al. 2004; Roehner 2007; Helmstetter and Sornette 2003; Helmstetter et al. 2003). Therefore, we suggest that the endo-exo framework provides a fruitful domain of investigation that may lead to new insighst in the dynamics of these systems.

It is interesting to mention that the question of exogenous versus endogenous forcing has been hotly debated in economics for decades. A prominent example is the 
theory of Schumpeter on the importance of technological discontinuities in economic history. Schumpeter argued that "evolution is lopsided, discontinuous, disharmonious by nature... studded with violent outbursts and catastrophes... more like a series of explosions than a gentle, though incessant, transformation" (Schumpeter 1939). Endogeneity versus exogeneity is also paramount in economic growth theory (Romer 1996).

\subsection{Epidemic model of social interactions by word-of-mouth}

A particularly useful insight on the endo-exo question is provided by the class of epidemic cascade models of social interactions (see Sornette 2005 and http://www.er. ethz.ch/essays/origins for reviews). This theoretical framework is reviewed in Helmstetter and Sornette (2002), Saichev and Sornette (2004, 2006a,b, 2007), and Saichev et al. (2005).

Let us consider an observable characterizing the activity of humans within a given social network of interactions. This activity can be the rate of visits or downloads on an internet website, the sales of a book or the number of newspaper articles on a given subject. We envision that the instantaneous activity results from a combination of external forces such as news and advertisement, and of social influences in which each past active individual may impregnate other individuals in her network of acquaintances with the desire to act. This impact of an active individual onto other humans is not instantaneous as people react at a variety of time scales. The time delays capture the time interval between social encounters, the maturation of the decision process which can be influenced by mood, sentiments, and many other factors and the availabilty and capacity to implement the decision. The contacts and exchanges between humans lead to information cascades.

This leads to identify a critical parameter, the branching ratio $n$, which controls the propensity for news, shocks, changes to propagate and percolate within the social network. When $n<1$, the social network is sub-critical and local changes remain localized. When $n$ reaches 1 , the system is critical, characterized by power laws in the distribution of group sizes impregnated by a given change. For $n>1$, the network is super-critical as local changes have a finite probability to propagate and invade the entire network. The regime $n \geq 1$ is the one of interest for marketing campaigns, for policy targets, more generally, for any action that aims at a maximum impact with a minimal cost. Typically, an external input of amplitude $S$ is amplified by the network effect of word-of-mouth epidemics by the factor $1 /(1-n)$ (for $n<1)$ (Helmstetter and Sornette 2002; Saichev and Sornette 2004, 2006a,b, 2007; Saichev et al. 2005), suggesting that policy making and marketing campaigns should not only optimize their action $S$ but also target mature and receptive networks characterized by a branching ratio close to, equal to or even larger than 1 . Concretely,

- In the commercial sale example, $S$ is the cost of an advertising campaign and $S /(1-n)$ is the average sale volume resulting from it.

- For financial crashes, $S$ could be the amplitude of the financial volatility shock resulting from exogenous events such as the coup against Gorbachev or 9/11, while $S /(1-n)$ is the cumulative volatility integrated over the whole future resulting from these shocks. 
- For social unrest, $S$ is the initial number of people involved in a social unrest, and $S /(1-n)$ is the total size of the population that ends up participating in the social upheaval.

- For earthquakes, $S$ is the rate of so-called background events resulting from the underlying plate tectonic motion, while $S /(1-n)$ is the total rate of seismicity triggered by these events.

These ideas and quantitative results are relevant to social unrest shocks, internal downloads, dialog in email traffic, the dynamics of commercial sale in response to advertisement or to word-of-mouth, volatility clustering and shocks in financial markets, dynamics of exploits and patches following disclosures of software vulnerability, movie blockbusters, the dynamics of YouTube video sharing, and so on. Our group is currently analyzing very large databases on software vulnerabilities and on YouTube video sharing (http://www.youtube.com), and preliminary results confirm the relevance of the endo-exo concepts as well as the existence of an ubiquitous power law response in time of these systems to shocks.

\subsection{A conjecture on happiness}

Recent works by Kahneman and others show that humans have a kind of reference point for happiness (Reichhardt 2006). This reference point may be different from one human being to the next. From two kinds of interviews, researchers have documented that humans are subjected to burst of happiness or despair which then relax after some time to their previous happiness level, similar to the relaxation of the response function due to an exogenous shock. Someone who gains a big lottery or someone who suffers a major accident leading to paralysis for instance will have a large instantaneous perturbation in their level of happiness, but remarkably both will return after some time and some adjustment to basically their previous level of happiness.

This suggests that happiness and well-being in humans can be approached by the generic endo-exo approach outlined in the previous section. The guideline offered by this insight is that we need to understand what are the individual, cultural and societal variables that may control the "criticality" parameter $n$ of our response to the incessant flux bathing our life, so that, under a constant background of "happiness sources" $S$, the overall happiness level is amplified to $S /(1-n)$ by internal and social cascades. This suggests to emphasize the policy impact on $n$ (our internal state) more than on $S$, the external sources of improvements. One of the interesting consequences of this dynamical view of happiness is to suggest the existence of a continuum between joy/sorrow (the instantaneous response to a source of pleasure/satisfaction versus a source of injury, loss or despair) and happiness/depression, mediated via the relaxation in time.

\section{Bubbles everywhere}

\subsection{Breakdown of "Galilean invariance principle" in human psychology}

I propose the idea that the collective dynamics of human affairs exhibit a breakdown of "Galilean invariance principle". In physics, Galilean Invariance is a principle of 
relativity which states that the fundamental laws of physics are the same in all inertial frames. Its direct consequence is that deviations of a body from constant velocity can only occur upon the application of a force. The analogy is that a constant velocity or rate of change is boring to the psychology of human beings, who tend to act to develop intermittent accelerating outcomes. In other words, my hypothesis is that the dynamics of human societies should be punctuated by series of accelerated bursts followed by corrections or phases of stasis.

A good example is found in financial markets, where bubbles and crashes are quite common. As a consequence of the individual actions of investors aggregated at the collective level, one can observe super-exponential acceleration of their processes punctuated by corrections and crashes (see Sornette 2003a,b and references therein). The perhaps most striking illustration is the market of Hong Kong, arguably until 1997 the freer market in the world with complete flexibility in the mobility of capital and its investment use. While the Hang Seng Hong Kong index from 1979 to 1997 is characterized by an approximately constant annual return of $14 \%$, this average return is a very poor description of what was actually occurring in the market: either it was super-exponentially accelerating, or it was crashing. This observation has been described and generalized to many bubbles and crashes in different parts of the world and at many epochs (see Sornette 2003a,b and references therein).

The underlying mechanism involves positive feedbacks on prices, i.e., the fact that, conditioned on the observation that the market has recently moved up (respectively down), this makes it more probable to keep it moving up (respectively down) in an amplified move, so that a large cumulative move ensues. The concept of "positive feedbacks" has a long history in economics and is related to the idea of "increasing returns"-which says that goods become cheaper the more of them are produced (and the closely related idea that some products, like fax machines, become more useful the more people use them). "Positive feedback" is the opposite of "negative feedback", a concept well-known for instance in population dynamics: the larger the population of rabbits in a valley, the less they have grass per rabbit. If the population grows too much, they will eventually starve, slowing down their reproduction rate which thus reduces their population at a later time. Thus negative feedback means that the higher the population, the slower the growth rate, leading to a spontaneous regulation of the population size; negative feedbacks thus tend to regulate growth towards an equilibrium.

In contrast, positive feedback asserts that the higher the price or the price return in the recent past, the higher will be the price growth in the future. Positive feedbacks, when unchecked, can produce runaways until the deviation from equilibrium is so large that other effects can be abruptly triggered and lead to rupture or crashes. The positive feedback leads to speculative trends which may dominate over fundamental beliefs and which make the system increasingly susceptible to any exogenous shock, thus eventually precipitating a crash. There are many mechanisms in the stock market and in the behavior of investors which may lead to positive feedbacks. They can be roughly divided into two classes.

- Technical and rational mechanisms for positive feedbacks:

1. Option hedging,

2. Insurance portfolio strategies, 
3. Trend following investment strategies,

4. Asymmetric information on hedging strategies.

- Behavioral mechanism for positive feedbacks based on

(a) The drive to actively escape boredom (the breakdown of "Galilean invariance principle" alluded to above),

(b) The psychology of imitation:

- Imitation is rational,

- Imitation is the highest cognitive task,

- We mostly learn by imitation,

- Imitation and "conventions" shape culture and social interactions.

Sornette (2003a,b) presents detailed documentation and arguments emphasizing the positive feedbacks by imitation, which is also known as the "herd" or "crowd" effect. Theoretical models implementing these ideas have been proposed by Ide and Sornette (2002) and by Sornette and Andersen (2002) and Andersen and Sornette (2004).

\subsection{Positive effect of ubiquitous "bubbles" in human affairs}

I propose to generalize the observation that financial markets exhibit alternating regimes of over-enthusiasm leading to bubbles followed by phases of consolidation, bearish trends or even crashes. In finance and economics, the term "bubble" refers to a situation in which excessive public expectations of future price increases cause prices to be temporarily elevated without justification from fundamental valuation. I extend this definition to human affairs as follows.

Definition of a "bubble" in human affairs: a "bubble" occurs when excessive public/political expectations of positive outcomes cause over-enthusiasm and unreasonable investment and efforts.

During bubbles, people take inordinate risks that would not otherwise be justified by standard cost-benefit and portfolio analysis. Instead, people rationalize their risktaking behavior by new models of net-present-value, such as witnessed during the new economy bubbles of IT and Internet companies that culminated in 2000: the new accounting method over-emphasized the "real option" value of companies associated with the new niches that they were opening. I want to emphasize the role of bubbles in human affairs because they seem inherently associated with the innovation process and the creation of new technology. Bubbles lead to a lot of destruction of value but also to the exploration and discovery of exceptional niches. Only during these times do people dare explore new opportunities, many of them unreasonable and hopeless, with rare emergences of great lucky outcomes. This is the wild risk regime of extremely heavy tails in the distribution of economic returns on investments. I envision this mechanism as the leading one controlling the appearance of disruptive innovations and major advances. In a word, bubbles (collective over-enthusiasm) seem a necessary evil to foster our collective attitude towards risk taking and break the stalemate of society resulting from its tendency towards risk avoidance. Bubbles are times of self-organized self-excited auto-catalytic amplification of risks that allow the exploration of new niches. I contend that society needs bubbles because the bubbles lead to a very risky 
behavior that lead to great potential returns. In absence of bubble psychology, no large risks are taken and no large return can derive leading to stagnation.

To illustrate this hypothesis, many examples can be put forward. Here, I discuss briefly only a few salient cases.

- Great boom of railway shares in Britain in the 1840s. Consider the great boom in railway shares in Britain in the 1840s. The boom and collapse not only depleted the wealth of many individuals, but cut briefly into the capital available for normal trade and finance. The overbuilding of railroads meant that few could earn back a return commensurate with the capital put into them.

Yet, Britain ended up with an extensive railway system ahead of other industrializing nations. Even if the building was done inefficiently, the gains to the economy were rather large and may have been part of the reasons that led to the great success of development of the United Kingdom in the Nineteen century (note that, at its apogee, the economic GDP under the influence of the UK amounted to almost two-third of the world output, compared to the apogee of the USA, which barely reached one-third of the world GDP a century later).

- Apollo program. On the fifth flight, Apollo 11 landed on the moon, 20 July 1969; Armstrong and Aldrin became the first humans to land and walk, at an estimated cost of 20-25 billion (of 1969 US dollars). At this time, what were the anticipations for the following 30 years, at the horizon of the new millenia? Great expectations included to put the Moon and Mars in "mankind's sphere of economic influence" (to use a phrase later chiseled by Presidential Science Advisor Jack Marburger). In the late 1960s, it was thought that permanent bases on the Moon would be routinely operated and that mankind would have already landed on Mars and beyond.

What is the state of space exploration and conquest in 2007? Would mankind be able to land again on the Moon and at what cost? Personally, as a teenager at the time of the historical landing who has become a grown-up over these more than 30 years, and as a scientist having contributed a bit to alleviate technical problems in components of spacecrafts (Anifrani et al. 1995, 1999; Maveyraud et al. 2001; Le Floc'h and Sornette 2003), I find the present situation on space exploration quite disappointing and depressing. It is clear that the expectations have been unfulfilled and that there are still major obstacles to overcome: protection of humans from cosmic rays, medical problems appearing in absence of gravity, reliability of spacecrafts, propulsion efficiency to cite a few.

One should balance this view by putting also the Apollo program in the context of the competition with the Soviets. There was certainly a large amount of propaganda press during the cold war, so that the actual reason for the moon landing program must be considered. In this respect, the huge costs for the moon landing may have actually provided a good return-on-investment as this project was one of several which locked the Soviets into an arms race. This race in conjunction with a number of issues with their internal political and economic system lead to the eventual collapse of the Soviet empire and to the victory of the US empire in the cold warexactly what was intended. But this is precisely the point of my hypothesis that bubbles are associated with large risks not otherwise embraced, which may lead to great returns. 
One can conclude conservatively that there is probably an entangled set of reasons that led to the "Apollo bubble". Beyond and aside of the complex problem of disentangling the origins and the mechanisms of the Apollo bubble, it remains evident that the returns-on-investment of space exploration from an industrial and economic view point are far from obvious. However, as vividly expressed by NASA administrator Michael Griffin (2007), space exploration is perhaps the best incarnation of the "real reasons" for taking risks in unreasonable (to standard cost/benefit analysis) endeavors. The "real reasons" that go beyond reason perhaps help define human beings. They include the enthusiasm for new things, the wonder and awe of discoveries, the challenges of competition, the taking of hard challenges... for the sake of the challenge.

- Human Genome project. "The ultimate goal of this initiative is to understand the human genome" (US Department of Energy, 1984, 1986). A 'rough draft' of the genome was finished in 2000 (announced jointly by then US president Bill Clinton and British Prime Minister Tony Blair on June 26, 2000). May 2006: sequence of the last chromosome for a total estimated cost of $\$ 3$ billion. The claims was/is that knowing the sequence of the genes would open immediately the door to great discoveries in medicine.

However, for any reasonable scientist acquainted with complex systems (and the human body and its immune system in particular are clearly very complex systems by all definitions of the term), it was clear that this was oversold: knowing the sequence of letters in a text without knowing the language brings little immediately and the major obstacles remain, which is that of understanding the text. This involves many many-body problems (since hundreds to thousands genes act often in concert and each may have several actions in multitude of diseases and expressions). For instance, using statistical and bioinformatic tools, Wood et al. (2007) have found recently that Human breast and colorectal cancers are linked with a much larger number of infrequent gene mutations that previously thought, rather than with large mutation frequencies of just a few genes.

But this "bubble" or enthusiasm on genomics has served its goal of promoting a branch of research, which is at its very beginning. This bubble led to large investments, large risks were taken, and little return has yet to be realized, except for some niches. It is interesting to read the statements of the community in the present post-bubble era, emphasizing that decades are needed to really exploit the data. In the same vein, the recent doubling of the USA effort in bio-medical research could be argued to be part of the same trend, whose fruits still have to grow and show their benefits to society (Check 2006). Of course, I am not arguing against the need for long-term investment in basic research, on the contrary, as can be seen from my own incursion in this debate mentioned above (Sornette and Zajdenweber 1999). It is true that the Human Genome project has led to a large boom for instance in Bioinformatics research and in genetic regulatory networks, which has benefitted greatly from the new data. It is probable that the Human Genome project has opened the door to great discoveries in medicine, but this is not in contradiction with my point, which is just to stress that the benefits will probably take much more time to come that expected when the bubble was inflating 
at full speed. I see this as somewhat similar to the railway boon in the UK in the nineteen century discussed above.

- Dolly the Sheep (5 July 1996, 14 February 2003). "Suspendous" and "mindboggling" were just two reactions to the birth of Dolly, the first mammal cloned from an adult cell. Suddenly, the idea of herds of identical prize bulls, or sheep producing medicines for humans in their milk, seemed wholly plausible. Then there was therapeutic cloning, which would provide genetically matched human tissue to patch up even the most seriously ill patient...

A New Scientist article in 2006 (Aldhous and Coghlan 2006) states: "Much of the excitement surrounding the creation of the first animal clone has vanished and therapeutic cloning is in the doldrums. But her influence should not be downplayed..." A report in Nature (Check 2007) confirms: “...these advances haven't led to big improvements in the cloning process, or yielded huge commercial payoffs."

- The IT and Internet bubble until March 2000. The so-called "new economy" bubble on information technology and the internet has been associated with huge investments (and big losses) on the IT sector. Few of the "dot-com" companies into which investors have poured billions of dollars of capital have lived to pay them an adequate return. Many companies have died but a few have survived and some have become giants (Yahoo, Google,...).

The world has also set the pace on trying out new Internet business models, and presumably will benefit enormously from the experience gained by the tens of thousands of engineers, entrepreneurs, and web designers who have acquired human capital in a new industry. Thus again, a bubble has left many "dead" but a few great innovations emerged, with the accumulation of new human capital.

We propose that these different examples are cases of positive feedbacks in action, which provide a common foundation to study these bubbles in human affairs.

How should this hypothesis on the "ubiquitous bubbles in human affairs" be tested? One would like a model with the relevant variables to express the feedback processes acting between and within human groups along the different hierarchical network structures of human society. The models could be developed at the macro, meso and/or micro levels. The macro level would involve aggregate variables and effective laws of feedback processes. The micro level should describe the preferences and behaviorial traits of individual agents and how they contribute and aggregate to the macro organization of bubbles in human affairs. The meso level could be a combination of the two. I believe that the models should be informed by the recent explosion of information on human cooperation, human competition, and human behavioral biases. While the relevant synthesis is in my mind not yet ripe, I hope to contribute in the future on this theme and perhaps to foster the interest of others.

\section{Illusion of control}

Human beings like to believe they are in control of their destiny. This ubiquitous trait seems to increase motivation and persistence, and is probably evolutionarily adaptive. The success of science and technology, with the development of ever more sophisticated computerized integrated sensors in the biological, environmental and 
social sciences, illustrate the quest for control as a universal endeavor. The exercise of governmental authority, the managing of the economy, the regulation of financial markets, the management of corporations, and the attempt to master natural resources, control natural forces and influence environmental factors all arise from this quest.

Langer (1975)'s phrase, "illusion of control" describes the fact that individuals appear hard-wired to over-attribute success to skill, and to underestimate the role of chance, when both are in fact present. Whether control is genuine or merely perceived is a prevalent question in psychological theories. outcomes, especially when they are the result of aggregations of individual optimization processes. But how good really is our ability to control? How successful is our track record in these areas? There is little understanding of when and under what circumstances we may over-estimate or even lose our ability to control and optimize.

Satinover and Sornette (2007a,b) have demonstrated analytically using the theory of Markov Chains and by numerical simulations in two classes of games, the Minority game and the Parrondo Games, that agents who optimize their strategy based on past information actually perform worse than non-optimizing agents. In other words, low-entropy (more informative) strategies under-perform high-entropy (or random) strategies. This provides a precise definition of the "illusion of control" in set-ups a priori defined to emphasize the importance of optimization.

Our robust message is that, under bounded rationality, the simple (large-entropy) strategies are often to be preferred over more complex elaborated (low-entropy) strategies. This is a message that should appeal to managers and practitioners, who are well-aware in their everyday practice that simple solutions are preferable to complex ones, in the presence of the ubiquitous uncertainty. More examples should be easy to find. For instance, control algorithms, which employ optimal parameter estimation based on past observations, have been shown to generate broad power law distributions of fluctuations and of their corresponding corrections in the control process, suggesting that, in certain situations, uncertainty and risk may be amplified by optimal control (Eurich and Pawelzik 2005). In the same spirit, more quality control in code development often decreases the overall quality which itself spurs more quality control leading to a vicious circle (Berczuk and Appleton 2002). In finance, there are many studies suggesting that most fund managers perform worse than random (Malkiel 2003) and strong evidence that over-trading leads to anomalously large financial volatility (Shiler 1992). Let us also mention the interesting experiments in which optimizing humans are found to perform worse than rats (Grandin and Johnson 2004). We conjecture that the illusion-of-control effect should be widespread in many strategic and optimization games and perhaps in many real life situations. This puts the question at a quantitative level so that it can be studied rigorously to help formulate better strategies and tools for management and policy, which take into account the intrinsic limitations of control in complex set-ups with feedbacks.

\section{Concluding remarks}

I have tried here to weave an outline of how the science of complexity, which includes evidence from both the natural and the social sciences, may help in addressing some of 
the most important as well as difficult question confronting mankind. In this journey, as can be expected, we find more questions than answers, more puzzles than solutions, more challenges than settlements.

Several other important ingredients have been left out. For instance, recent research in cognitive sciences and in anthropology suggest that human beings are made to interact with no more than about 150 other humans (Dunbar 1998), otherwise conflicts arise and fragmentation follows. Actually, carefully studies of human groups show the existence of a delicate hierarchy of natural group sizes (Zhou et al. 2005), that may be a result of evolution. This suggests that the coordination of human activities in large modern society needs to recognize this essential cognitive limitation probably deeply rooted in our emotional and rational brain.

Another ingredient which in my mind needs to be incorporated in a science-based approach to policy decision making is the context-dependent, cultural as well as evolutionary control of human cooperation, based on the existence of feedbacks such a reward and punishments (Fehr and Fischbacher 2003; Darcet and Sornette 2008). The fundamental question here is to identify the springs at the origin of cooperation and the elements (structural and dynamical) that may hinder or destroy it.

Acknowledgments This paper was prepared as an invited talk for the workshop Trans-disciplinary Research Agenda for Societal Dynamics (http://www.uni-lj.si/trasdinLjubljana), organized by Professor J. Rogers Hollingsworth, Dr. Karl H. Mueller, Professor Ivan Svetlik (vice rector of the University of Ljublana), 24-25 May 2007, Ljubljana, Slovenia. I am grateful for the feedbacks offered by the participants.

\section{References}

Aldhous P, Coghlan A (2006) Dolly's cloning revolution fails to materialise. New Sci 2558:8

Andersen JV, Sornette D (2004) Fearless versus fearful speculative financial bubbles. Physica A 337(3-4): $565-585$

Anifrani J-C, Le Floc'h C, Sornette D, Souillard B (1995) Universal log-periodic correction to renormalization group scaling for rupture stress prediction from acoustic emissions. J Phys I France 5(6):631-638

Anifrani J-C, Le Floc'h C, Sornette D (1999) Prédiction de la rupture de réservoirs composites de haute pression à l'aide de l'émission acoustique, Contrôle Industriel 220:43-45

Bak P (1996) How nature works: the science of self-organized criticality. Copernicus, New York

Bak P, Paczuski M (1995) Complexity, contingency, and criticality. Proc Natl Acad Sci USA 92:6689-6696

Berczuk SP, Appleton B (2002) In software configuration management patterns: effective teamwork, practical integration. Addison-Wesley, Reading

Buchanan M (1996) Measuring science revolutions. Nature 384:325

Check E (2006) Biomedical research: facing the opposition. Nature 441:17-19. doi:10.1038/441017a

Check E (2007) Cloning special: Dolly: a hart act to follow. Nature 445:802. doi:10.1038/445802a

Crane R, Sornette D (2008) Robust dynamic classes revealed by measuring the response function of a social system, preprint http://arXiv.org/abs/0803.2189

Darcet D, Sornette D (2008) Quantitative determination of the level of cooperation in the presence of punishment in three public good experiments. J. Econ Interac Coord. doi:10.1007/s11403-007-0027-X

Deschatres F, Sornette D (2005) The dynamics of book sales: endogenous versus exogenous shocks in complex networks. Phys Rev E 72:016112

Dieks and Chang (1976) Differences in impact of scientific publications: some indices derived from a citation analysis. Soc Stud Sci 6:247

Dunbar RIM (1998) The social brain hypothesis. Evol Anthrop 6:178-190

Ehrenreich H (1995) Strategic curiosity: Semiconductor physics in the 1950s. Phys Today January:28-34

Einstein A (1905) Über die von der molekularkinetischen Theorie der Wärme geforderte Bewegung von in ruhenden Flüssigkeiten suspendierten Teilchen. Ann Phys 17:549 
Einstein A (1956) Investigations on the Theory of Brownian Movement. Dover, New York

Eurich CW, Pawelzik K (2005) In artificial neural networks: formal models and their applications-ICANN 2005. Springer, Berlin, p 0302

Fehr E, Fischbacher U (2003) The nature of human altruism. Nature 425:785-791

Grabowski HG, Vernon JM (1990) A new look at the returns and risks to pharmaceutical R\&D. Management Sci 36:804-821

Grabowski HG, Vernon JM (1994) Returns to R\&D on new drug introductions in the 1980s. J Health Econ 13:383-406

Grandin T, Johnson C (2004) Animals in translation. Scribner, New York

Griffin J (2007) What do happiness studies study? J Happiness Stud. doi:10.1007/s10902-006-9007-4

Griffin M (2007) Space exploration: real reasons and acceptable reasons, Comments made at the Quasar Award Dinner, Bay Area Houston Economic Partnership on 19 Jan 2007. http://www.spaceref.com/ news/viewsr.html?pid=23189

Helmstetter A, Sornette D (2002) Sub-critical and supercritical regimes in epidemic models of earthquake aftershocks. J Geophys Res 107(B10): 2237. doi:10.1029/2001JB001580

Helmstetter A, Sornette D (2003) Foreshocks explained by cascades of triggered seismicity. J Geophys Res (Solid Earth) 108(B10):2457. doi:10.1029/2003JB00240901

Helmstetter A, Sornette D, Grasso J-R (2003) Mainshocks are Aftershocks of Conditional Foreshocks: How do foreshock statistical properties emerge from aftershock laws. J Geophys Res 108(B10):2046. doi:10.1029/2002JB001991

Holling CS, Gunderson L, Ludwig D (2002) In quest of a theory of adaptive change. In: Gunderson LH, Holling CS (eds) Panarchy: Understanding Transformations in Human and Natural Systems. Island Press, Washington, D.C. pp 3-24

Hollingsworth R, Hollingsworth EJ (2000) Major discoveries and biomedical research organizations: perspectives on interdisciplinarity, nurturing leadership, and integrated structure and cultures. In: Peter Weingart, Nico Stehr (eds) Practising interdisciplinarity, University of Toronto Press, Toronto, pp 215-244

Ide K, Sornette D (2002) Oscillatory finite-time singularities in finance, population and rupture. Physica A 307(1-2):63-106

Johansen A, Sornette D (2001) Large stock market price drawdowns are outliers. J Risk 4(2):69-110, Winter 2001/02. http://arXiv.org/abs/cond-mat/0010050

Johansen A, Sornette D (2007) Shocks, crash and bubbles in financial markets. Brussels Econ Rev Nonlinear Financ Anal, 149-2/Summer. http://arXiv.org/abs/cond-mat/0210509 (in press )

Kahneman D, Krueger AB, Schkade D, Schwarz N, Stone AA (2006) Would you be happier if you were richer, a focusing illusion. Science 312:1908-1910

Kuhn T (1970) The Structure of Scientific Revolutions, 2nd edn. University of Chicago Press, Chicago

Langer EJ (1975) J Pers Soc Psychol 32(2):311-328

Le Floc'h C, Sornette D (2003) Predictive acoustic emission: Application on helium high pressure tanks, Prédiction des évènements catastrophiques: une nouvelle approche pour le controle de santé structurale, vol 3(1-2). Instrumentation Mesure Metrologie (published by Hermes Science) RS serie I2M, pp 89-97

Malkiel BG (2003) A random walk down wall street:: the time-tested strategy for successful investing. WW Norton \& Company, New York

Martin Ben R et al (1996) The relationship between publicly funded basic research and economic performance, Report of the Science Policy Research Unit of the University of Sussex

Maveyraud C, Vila JP, Sornette D, Le Floch C, Dupillier JM, Salomé R (2001) Numerical modelling of the behaviour of high-pressure vessels under a hypervelocity impact. Mec Ind 2:57-62

National Academy of Sciences (1997) National Academy of Engineering, Institute of Medecine and National Research Council, Preparing for the 21st Century. http://www2.nas.edu/21st/

Osberg L, Sharpe A (2005) How should we measure the 'economic' aspects of well-being. Rev Income Wealth 51(2):311-336

Redner S (1998) How popular is your paper? An empirical study of the citation distribution. Euro Phys J B 4:131-134

Reichhardt T (2006) A measure of happiness. Nature 444:418-419

Revkin AC (2005) A new measure of well-being from a happy little kingdom. NY Times, October 4

Roehner BM (2007) driving forces in physical, biological and socio-economic phenomena: a network science investigation of social bonds and interactions, Cambridge University Press, London 
Roehner BM, Sornette D, Andersen JV (2004) Response functions to critical shocks in social sciences: an empirical and numerical study. Int J Mod Phys C 15(6):809-834

Romer D (1996) Advanced macroeconomics. McGraw-Hill, New York

Ruelle D (2004) Conversations on nonequilibrium physics with an extraterrestrial. Phys Today 57(5):48-53

Saichev A, Helmstetter A, Sornette D (2005) Anomalous scaling of offspring and generation numbers in branching processes. Pure Appl Geophys 162:1113-1134

Saichev A, Sornette D (2004) Anomalous power law distribution of total lifetimes of aftershock sequences. Phys Rev E 70:046123

Saichev A, Sornette D (2006a) Power law distribution of seismic rates: theory and data. Eur Phys J B 49:377-401

Saichev A, Sornette D (2006b) “Universal” distribution of inter-earthquake times explained. Phys Rev Lett 97:078501

Saichev A, Sornette D (2007) Theory of earthquake recurrence times. J Geophys Res 112:B04313, doi:10.1029/2006JB004536

Satinover JB, Sornette D (2007a) Illusion of control in minority and parrondo games. Eur Phys J B 60:369_ 384

Satinover JB, Sornette D (2007b) Illusion of control in a brownian game. Physica A 386:339-344

Scherer FM (1998) The size distribution of profits from innovation. Annale dEconomie et de Statistique 49(50):495-516

Schumpeter JA (1939) Business cycles: a theoretical, historical and statistical analysis of the capitalist process. McGraw-Hill, New York

Shiller RJ (1992) Market Volatility. MIT Press, Cambridge

Sornette D. (2002a) Economy of scales in innovations with block-busters. Quant Finance 2:224-227

Sornette D (2002b) Predictability of catastrophic events: material rupture, earthquakes, turbulence, financial crashes and human birth. Proc Natl Acad Sci USA 99(Supp 1):2522-2529

Sornette D (2003a) Why stock markets crash (critical events in complex financial systems). Princeton University Press, Princeton

Sornette D (2003b) Critical market crashes. Phys Rep 378(1):1-98

Sornette D (2004) Critical phenomena in natural sciences, chaos, fractals, self-organization and disorder: concepts and tools, 2nd edn. Springer Series in Synergetics, Heidelberg

Sornette D (2005) Endogenous versus exogenous origins of crises. In: Albeverio S, Jentsch V, Kantz H (eds) Extreme events in nature and society series: the frontiers collection. Springer, Heidelberg. http:// arxiv.org/abs/physics/0412026

Sornette D, Andersen JV (2002) A nonlinear super-exponential rational model of speculative financial bubbles. Int J Mod Phys C 13(2):171-188

Sornette D, Zajdenweber D (1999) The economic return of research: the Pareto law and its implications. Eur Phys J B 8(4):653-664

Sornette D, Malevergne Y, Muzy JF (2003) Tail risk: what causes crashes? Risk 16(2):67-71. http://arXiv. org/abs/cond-mat/0204626

Sornette D, Deschatres F, Gilbert T, Ageon Y (2004a) Endogenous versus exogenous shocks in complex networks: an empirical test using book sale ranking. Phys Rev Lett 93(22):228701

Sornette D, Malevergne Y, Muzy J-F (2004b) Volatility fingerprints of large shocks: endogenous versus exogenous. In: Takayasu H (ed) Application of econophysics. Proceedings of the second Nikkei symposium on econophysics. Springer, Berlin, ISBN 4-431-14028-X, 334 p 137 illus

Sornette D, Yukalov VI, Yukalova EP, Henry J-Y, Schwab D, Cobb JP (2007) Endogenous versus exogenous origins of diseases. J Theor Biol. http://arxiv.org/abs/0710.3859 (submitted)

Stratonovich RL (1992) Nonlinear nonequilibrium thermodynamics i: linear and nonlinear fluctuationdissipation theorems. Springer, Berlin

Zhou W-X, Sornette D, Hill RA, Dunbar RIM (2005) Discrete hierarchical organization of social group sizes. Proc R Soc London 272:439-444 